\title{
Effect of dihydropyrimidine dehydrogenase single nucleotide polymorphisms on prognosis of breast cancer patients with chemotherapy
}

\author{
Fengxia Qin ${ }^{1,3,4,5,6, *}$, Huikun Zhang1,3,4,5,6,*, Yong Huang $2,3,4,5,6, *$, Limin Yang $2,3,4,5,6$, \\ Feng $\mathbf{Y u}^{2,3,4,5,6}$, Xiaoli Liu' ${ }^{2,3,4,5,6}$, Li Fu' ${ }^{1,3,4,5,6}$, Feng Gu${ }^{1,3,4,5,6}$ and Yongjie Ma ${ }^{2,3,4,5,6}$ \\ ${ }^{1}$ Department of Breast Cancer Pathology and Research Laboratory, Tianjin Medical University Cancer Institute and Hospital, \\ Tianjin, China \\ ${ }^{2}$ Department of Tumor Cell Biology, Tianjin Medical University Cancer Institute and Hospital, Tianjin, China \\ ${ }^{3}$ National Clinical Research Center for Cancer, Tianjin, China \\ ${ }^{4}$ Tianjin's Clinical Research Center for Cancer, Tianjin, China \\ ${ }^{5}$ Key Laboratory of Cancer Prevention and Therapy, Tianjin, China \\ ${ }^{6}$ Key Laboratory of Breast Cancer Prevention and Therapy, Tianjin Medical University, Ministry of Education, Tianjin, China \\ * These authors have contributed equally to this work \\ Correspondence to: Yongjie Ma, email: yongjiemagu@aliyun.com \\ Keywords: DPYD; fluoropyrimidine; breast cancer; prognosis; chemotherapy \\ Received: June 20,2017 Accepted: November 26, $2017 \quad$ Published: December 08, 2017 \\ Copyright: Qin et al. This is an open-access article distributed under the terms of the Creative Commons Attribution License 3.0 \\ (CC BY 3.0), which permits unrestricted use, distribution, and reproduction in any medium, provided the original author and source \\ are credited.
}

\section{ABSTRACT}

Defining biomarkers that predict therapeutic effects and adverse events is a crucial mandate to guide patient selection for personalized cancer treatments. DPD (dihydropyrimidine dehydrogenase, encoded by DPYD gene) is the initial and ratelimiting enzyme of metabolic pathway of fluoropyrimidines, and fluoropyrimidines are common used drug therapies for breast cancer. Previous studies on DPYD polymorphism were mainly focused on its association with fluoropyrimidines toxicity. In our present study, 5 DPYD single nucleotide polymorphisms status was detected from tumor tissues of $\mathbf{3 3 1}$ invasive breast cancer patients using standard techniques. We for the first time investigated the prognostic significance of DPYD polymorphisms in breast cancer. We demonstrated non-luminal breast cancer patients carrying DPYD c.1627A>G AG/GG treated with fluoropyrimidine-based regimen presented a shorter overall survival and progression-free survival than carriers treated with nonfluoropyrimidine regimen. However, non-luminal DPYD c.1627A>G AG/GG carriers treated with TE (taxane and anthracycline)-based regimen showed a better prognosis compared with carriers treated with non-TE regimen. Our results suggested TE-based chemotherapy was a suitable regimen for non-luminal patients with $D P Y D$ c.1627A>G AG/GG genotype and fluoropyrimidine-based regimen should not be recommended for those patients. Our findings provided a novel strategy, which will guide clinicians to choose more precise chemotherapy treatment for breast cancer patients.

\section{INTRODUCTION}

Precision medicine in oncology is the result of an increasing awareness of patient specific clinical features. It does not only mean utilization of tumor biomarkers, but also relevant germline-mutation-detections in drug metabolizing enzymes and transporters, which have been shown to impact drug response, providing rationale for individualized treatment [1].

Fluoropyrimidines are the most prevalent and effective chemotherapeutic agents used for the systemic treatment of various malignancies including 


\begin{tabular}{|c|c|}
\hline PCR reaction & Primer sequences \\
\hline \multicolumn{2}{|c|}{ c. $74 \mathrm{~A}>\mathrm{G}$ and $\mathrm{c.85T}>\mathrm{C}$} \\
\hline forward & 5'-GCAGTGAACTGAGATTGTACCACT-3' \\
\hline reverse & 5'-CTTGCCTTACAATGTGTGGAG-3' \\
\hline \multicolumn{2}{|l|}{ c. $1627 A>G$} \\
\hline forward & 5'-TATTATATGGACAATTTAGAT-3' \\
\hline reverse & 5'-GATAGACATTTCTATATGACT-3' \\
\hline \multicolumn{2}{|l|}{ c. $1896 \mathrm{~T}>\mathrm{C}$} \\
\hline forward & 5'-TCATCAGGACATTGTGACAAAT-3' \\
\hline reverse & 5'-СТTTCTATGCATCAGCAAAGC-3' \\
\hline \multicolumn{2}{|l|}{ c. $2194 \mathrm{G}>\mathrm{A}$} \\
\hline forward & 5'-GTAGGAGTTAAATTAGTGAAG-3' \\
\hline reverse & 5'-AGCAACСТCCAAGAAAGCACA-3' \\
\hline
\end{tabular}

gastrointestinal, breast, pancreas and head and neck cancers [2-5]. DPD (dihydropyrimidine dehydrogenase), encoded by DPYD gene, is the initial and rate-limiting enzyme of the metabolic pathway of fluoropyrimidines, such as 5-Fu, capecitabine and tegafur [6-8]. The clinical importance of DPD was initially identified due to severe or lethal toxicity in patients given fluoropyrimidines who are deficient in or have low levels of DPD activity [9-11]. Since then, more than 50 DPYD polymorphisms have been reported to cause fluoropyrimidine-associated toxicity in the treatment of malignancies such as colorectal carcinoma, gastroesophageal cancer and lymphoblastic leukemia [12-14]. Recently, emerging evidence indicated that DPYD polymorphisms could contribute to tumorgenesis and influence the chemosensitivity as well as clinical outcomes of cancer patients. It was reported that DPYD single nucleotide polymorphisms (SNPs) led to an increased risk of ovarian cancer and gastrointestinal tumors patients with $D P Y D$ c. $1627 \mathrm{~A}>\mathrm{G}$ AG/GG genotype presented low chemosensitivity to fluorouracil-based adjuvant treatment $[15,16]$. Furthermore, DPYD SNPs (rs1760217) were significantly associated with reduced survival in pancreatic cancer patients [17]. However, the prognostic significance of DPYD polymorphisms in breast cancer has rarely been investigated.

In our present study, 5 DPYD SNPs status (c.74A $>\mathrm{G}$, c. $85 \mathrm{~T}>\mathrm{C}$, c. $1627 \mathrm{~A}>\mathrm{G}$, c. $1896 \mathrm{~T}>\mathrm{C}$, c. $2194 \mathrm{G}>\mathrm{A}$ ) were detected in tumor tissues from 331 invasive breast cancer patients. We demonstrated for the first time that DPYD SNPs status was associated with breast cancer prognosis, especially the impact of c. $1627 \mathrm{~A}>\mathrm{G}$ polymorphism on prognosis of non-luminal subtype. We found that nonluminal breast cancer patients carrying $D P Y D$ c. $1627 \mathrm{~A}>\mathrm{G}$ AG/GG genotype treated with fluoropyrimidine-based regimen presented a shorter overall survival (OS) and progression-free survival (PFS) compared with carriers treated with non-fluoropyrimidine regimen. However, non-luminal DPYD c.1627A > G AG/GG genotype carriers treated with TE (taxane and anthracycline)-based regimen showed a better prognosis compared with carriers treated with non-TE regimen. All these results suggested that TE-based chemotherapy was a suitable regimen for nonluminal breast cancer patients with DPYD c.1627A $>\mathrm{G} \mathrm{AG} /$ GG genotype and fluoropyrimidine-based chemotherapy should not be recommended for these patients. Our findings provided a novel strategy, which will guide clinicians to choose more precise chemotherapy treatment for breast cancer patients.

\section{RESULTS}

\section{Association between DPYD SNPs status and prognosis of patients with fluoropyrimidine- based chemotherapy, especially in non-luminal subtype breast cancer}

Primers for 5 DPYD SNPs amplifications were presented in Table 1 and genotypic frequencies and characteristics of 331 breast cancer specimens were shown in Table 2. In this study, c.74A $>$ G and c.2194G $>$ A SNPs were excluded due to a limited frequency (minor allele frequency $<5 \%$ ). The observed genotype frequencies of c. $85 \mathrm{~T}>\mathrm{C}$, c. $1627 \mathrm{~A}>\mathrm{G}$ and c. $1896 \mathrm{~T}>\mathrm{C}$ were all in HardyWeinberg equilibrium and they were analyzed in the following studies. Example sequence traces of DPYD SNPs were shown in Supplementary Figure 1.

We found that non-wild type DPYD (patients with c.85T $>$ C TT, c. $1627 \mathrm{~A}>\mathrm{G}$ AA and c.1896T $>$ C TT simultaneously were identified as wild type $D P Y D$ group, whereas patients with whichever of mutations at c.85T $>C$, 
Table 2: DPYD SNPs information and genotypic frequencies

\begin{tabular}{|c|c|c|c|c|}
\hline Genotype & Location & Effect & Cases $(\%)$ & HWE $P$ value \\
\hline c.85T $>C$ & Exon 2 & Cys29Arg & & 0.201 \\
\hline TT & & & 273 (82.5) & \\
\hline TC & & & $53(16.0)$ & \\
\hline $\mathrm{CC}$ & & & $5(1.5)$ & \\
\hline c. $1627 \mathrm{~A}>\mathrm{G}$ & Exon 13 & Ile543Val & & 0.144 \\
\hline AA & & & $193(58.3)$ & \\
\hline AG & & & $113(34.1)$ & \\
\hline GG & & & $25(7.6)$ & \\
\hline c. $1896 \mathrm{~T}>\mathrm{C}$ & Exon 14 & Phe632Phe & & 0.161 \\
\hline TT & & & $256(77.3)$ & \\
\hline TC & & & $67(20.3)$ & \\
\hline $\mathrm{CC}$ & & & $8(2.4)$ & \\
\hline c. $2194 \mathrm{G}>\mathrm{A}$ & Exon 18 & Val732Ile & & 0.824 \\
\hline GG & & & 323 (97.6) & \\
\hline GA & & & $8(2.4)$ & \\
\hline $\mathbf{A A}$ & & & $\mathbf{0}(0)$ & \\
\hline c. $74 \mathrm{~A}>\mathrm{G}$ & Exon 2 & His25Arg & & $<0.001$ \\
\hline $\mathbf{A A}$ & & & $328(99.1)$ & \\
\hline GA & & & O (0) & \\
\hline GG & & & $3(1.6)$ & \\
\hline
\end{tabular}

HWE: Hardy-Weinberg equilibrium.

${ }^{\text {aTwo-sided }} \chi^{2}$ test.

c. $1627 \mathrm{~A}>\mathrm{G}$ or c. $1896 \mathrm{~T}>\mathrm{C}$ were identified as non-wild type $D P Y D$ group) was not correlated with clinicopathological characteristics (Supplementary Table 1).

To investigate the influence of DPYD SNPs on breast cancer prognosis, we compared the clinical outcome of patients with wild type or non-wild type DPYD and no obvious difference was found between them in OS analysis $(P=0.848$, Figure 1$)$. However, non-wild type $D P Y D$ carriers treated with fluoropyrimidine-based regimen exhibited a shorter OS compared with carriers treated with non-fluoropyrimidine regimen $(P=0.017$, Figure $2 \mathrm{~A}$ and $2 \mathrm{~B}$ ). We did not find any statistical difference in clinicopathologic characteristics between the two populations at baseline (Supplementary Table 2). Meanwhile, for wild type $D P Y D$ carriers, the clinical outcome of patients treated with fluoropyrimidinebased regimen was similar to carriers treated with non-fluoropyrimidine regimen (Figure 2C and 2D). It suggested that SNPs status of DPYD was associated with effect of fluoropyrimidine-based treatment.

Subsequently, the clinical significance of $D P Y D$ SNPs in different breast cancer molecular subtypes was examined. In non-luminal subgroup, non-wild type $D P Y D$ carriers treated with fluoropyrimidine-based regimen exhibited a worse prognosis compared with carriers treated with non-fluoropyrimidine regimen (Figure 3A-3D). We did not find any statistical difference in clinicopathologic characteristics between the two populations at baseline (Supplementary Table 3). However, in luminal subgroup, patients treated with fluoropyrimidine-based regimen exhibited a similar outcome compared with those treated with non-fluoropyrimidine regimen, regardless of their DPYD SNPs status (wild type or non-wild type) (Figure $3 \mathrm{E}-3 \mathrm{H}$ ). It suggested that non-luminal patients with non-wild type $D P Y D$ were unable to benefit from fluoropyrimidine-based chemotherapy.

\section{Association between c.1627A $>$ G AG/GG and prognosis of patients with fluoropyrimidine- based chemotherapy, especially in non-luminal subtype breast cancer}

In the following studies, we started to focus on the clinical significance of 3 polymorphisms, individually. 
For c. $1627 \mathrm{~A}>\mathrm{G}$ AG/GG genotype carriers, patients treated with fluoropyrimidine-based regimen exhibited a shorter OS and a tendency of shorter PFS compared with those treated with non-fluoropyrimidine regimen (Figure 4A and 4B). We did not find any difference in clinicopathologic characteristics between two populations at baseline (Supplementary Table 4). However, for c.1896T $>$ C TC/CC genotype carriers, patients treated with fluoropyrimidine-based regimen exhibited a similar OS and PFS compared with those treated with non-fluoropyrimidine regimen (Figure 4C and 4D). For c.85T $>\mathrm{C} \mathrm{TC} / \mathrm{CC}$ genotype carriers, patients treated with fluoropyrimidine-based regimen exhibited a similar OS and a shorter PFS compared with those treated with nonfluoropyrimidine regimen (Figure $4 \mathrm{E}$ and $4 \mathrm{~F}$ ). These results indicated that patients with c. $1627 \mathrm{~A}>\mathrm{G}$ AG/GG genotype could not benefit from fluoropyrimidine-based regimen.

Next, in-depth analysis was performed to identify which molecular subtype patients with c. $1627 \mathrm{~A}>\mathrm{G} \mathrm{AG} /$ GG genotype could not benefit from fluoropyrimidinebased regimen. For non-luminal subtype, c.1627A $>\mathrm{G}$ AG/GG genotype carriers treated with fluoropyrimidinebased regimen showed a shorter OS and PFS compared with carriers treated with non-fluoropyrimidine regimen (Figure 5A and 5B). We did not find any difference in clinicopathologic characteristics between two populations at baseline (Supplementary Table 5). Meanwhile, c.1896T $>$ C TC/CC genotype carriers treated with fluoropyrimidine-based regimen exhibited a similar OS and PFS (Figure 5C and 5D) compared with those treated

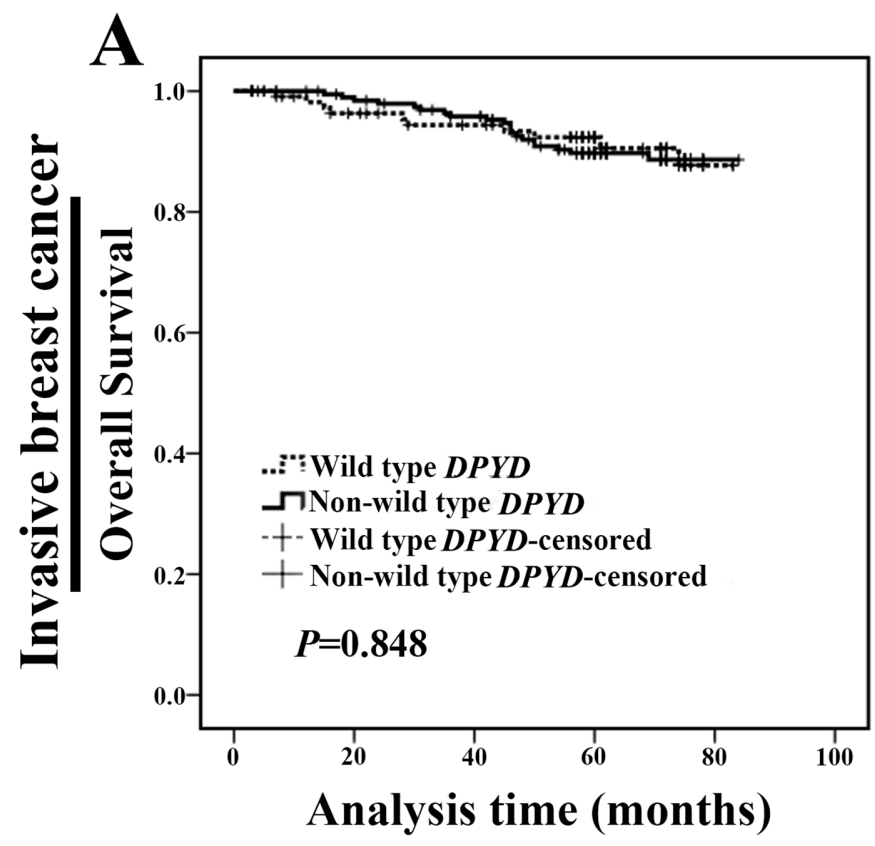

with non-fluoropyrimidine regimen; and c.85T $>\mathrm{C} \mathrm{TC/}$ CC genotype patients treated with fluoropyrimidinebased regimen exhibited a similar prognosis compared with carriers treated with non-fluoropyrimidine regimen (Figure 5E and 5F). These results indicated that nonluminal subtype patients with c. $1627 \mathrm{~A}>\mathrm{G}$ AG/GG could not benefit from fluoropyrimidine-based chemotherapy. Notably, luminal c.1627A $>$ G AG/GG carrier treated with fluoropyrimidine-based regimen exhibited a similar prognosis compared with carriers treated with nonfluoropyrimidine regimen (OS: $P=0.888$, PFS: $P=0.718$; Supplementary Figure 2).

\section{Association between c.1627A $>$ G AG/GG and prognosis of patients with TE-based chemotherapy, especially in non-luminal subtype breast cancer}

Besides fluoropyrimidine-based chemotherapy, TE (taxane and anthracycline) based therapies have been applied widely as the first-line treatment of breast cancer [18]. In the following experiments, we examined the relationship between DPYD SNPs and the effect of TE-based therapies. We found that non-wild type DPYD carriers treated with TE-based regimen exhibited a longer OS compared with carriers treated with non-TE regimen (Figure 6A and 6B). We did not find any statistical difference in clinicopathologic characteristics between the two populations at baseline (Supplementary Table 6). Meanwhile, for wild type DPYD carriers, the clinical outcome of patients treated with TE-based regimen was

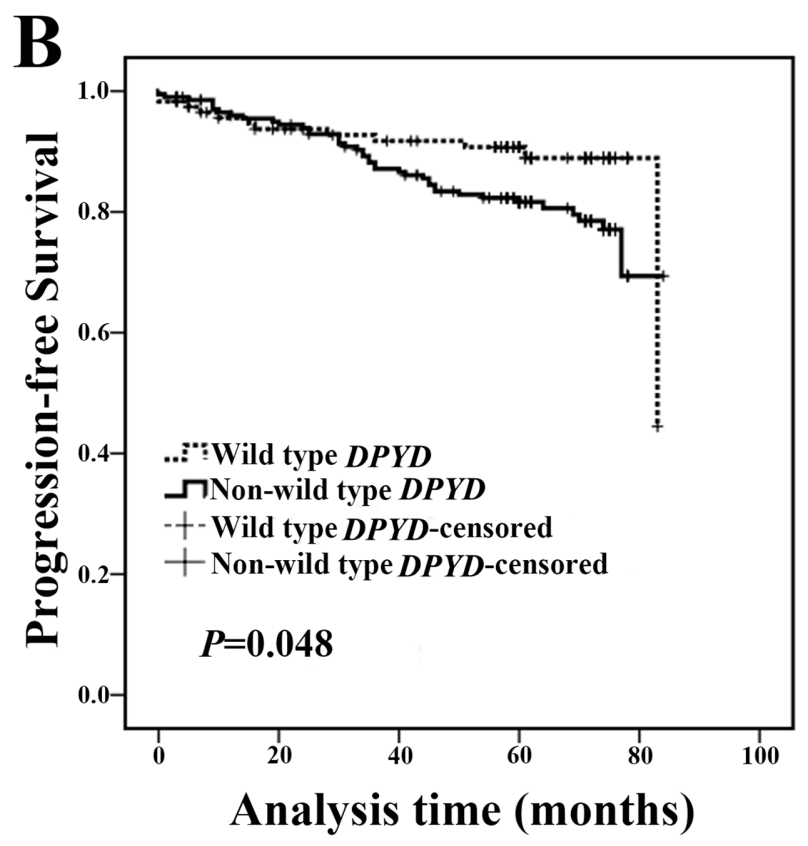

Figure 1: Relationship between DPYD SNPs status and breast cancer patients prognosis. (A) Patients with non-wild type $D P Y D$ exhibited a similar overall survival (OS) compared with wild type DPYD carriers (log-rank test). (B) Patients with non-wild type $D P Y D$ exhibited a shorter progression-free survival (PFS) compared with wild type DPYD carriers. 
similar to those treated with non-TE regimen (Figure 6C and 6D). These results suggested that breast cancer patients with non-wild type $D P Y D$ were more sensitive to TE-based chemotherapy.

Subsequently, the clinical significance of 3 polymorphisms was investigated, individually. For c. $1627 \mathrm{~A}>\mathrm{G}$ AG/GG genotype carriers, patients treated with TE-based regimen exhibited a longer OS compared with those treated with non-TE regimen (Figure 7A and 7B). We did not find any difference in clinicopathologic characteristics between two populations at baseline (Supplementary Table 7). Meanwhile, patients treated with TE-based regimen exhibited a similar prognosis with those treated with non-TE regimen in both c.1896T $>\mathrm{C}$ TC/CC genotype carriers and c.85T $>\mathrm{C} \mathrm{TC} / \mathrm{CC}$ genotype carriers (Figure 7C-7F).
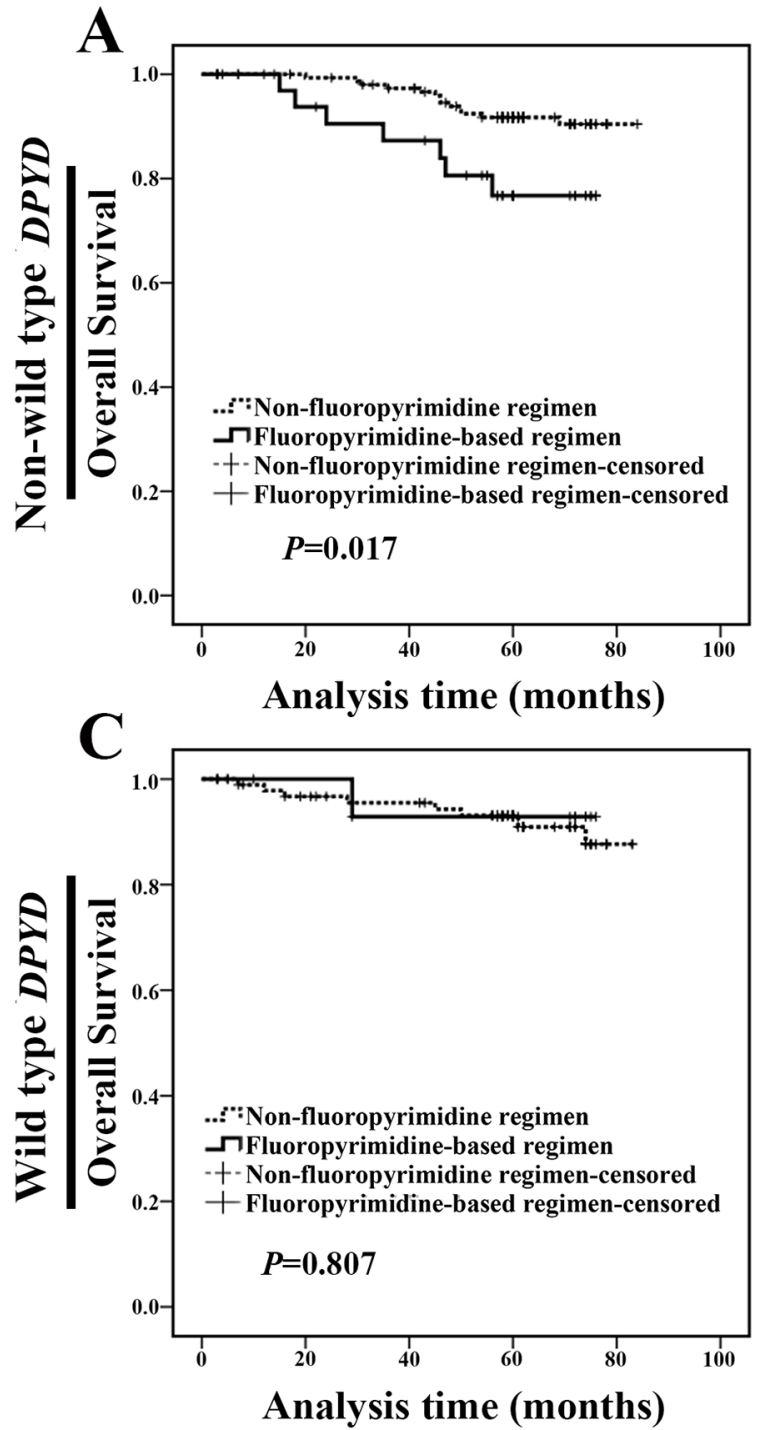

Furthermore, for non-luminal subtype patients with c. $1627 \mathrm{~A}>\mathrm{G}$ AG/GG genotype, those treated with TE-based regimen exhibited a better OS compared with patients treated with non-TE regimen (Figure $8 \mathrm{~A}$ and $8 \mathrm{~B}$ ). We did not find any difference in clinicopathologic characteristics between such two populations at baseline (Supplementary Table 8). For both non-luminal c.1896T $>\mathrm{C} \mathrm{TC/CC}$ and c. $85 \mathrm{~T}>\mathrm{C} \mathrm{TC} / \mathrm{CC}$ genotype carriers, patients treated with TE-based regimen exhibited similar prognosis compared with those treated with non-TE regimen (Figure $8 \mathrm{C}$ 8F). Meanwhile, luminal carriers treated with TE-based regimen exhibited similar OS compared with those treated with non-TE regimen (Supplementary Figure 3). These results indicated that non-luminal subtype patients with c. $1627 \mathrm{~A}>\mathrm{G}$ AG/GG could benefit from TE-based chemotherapy.
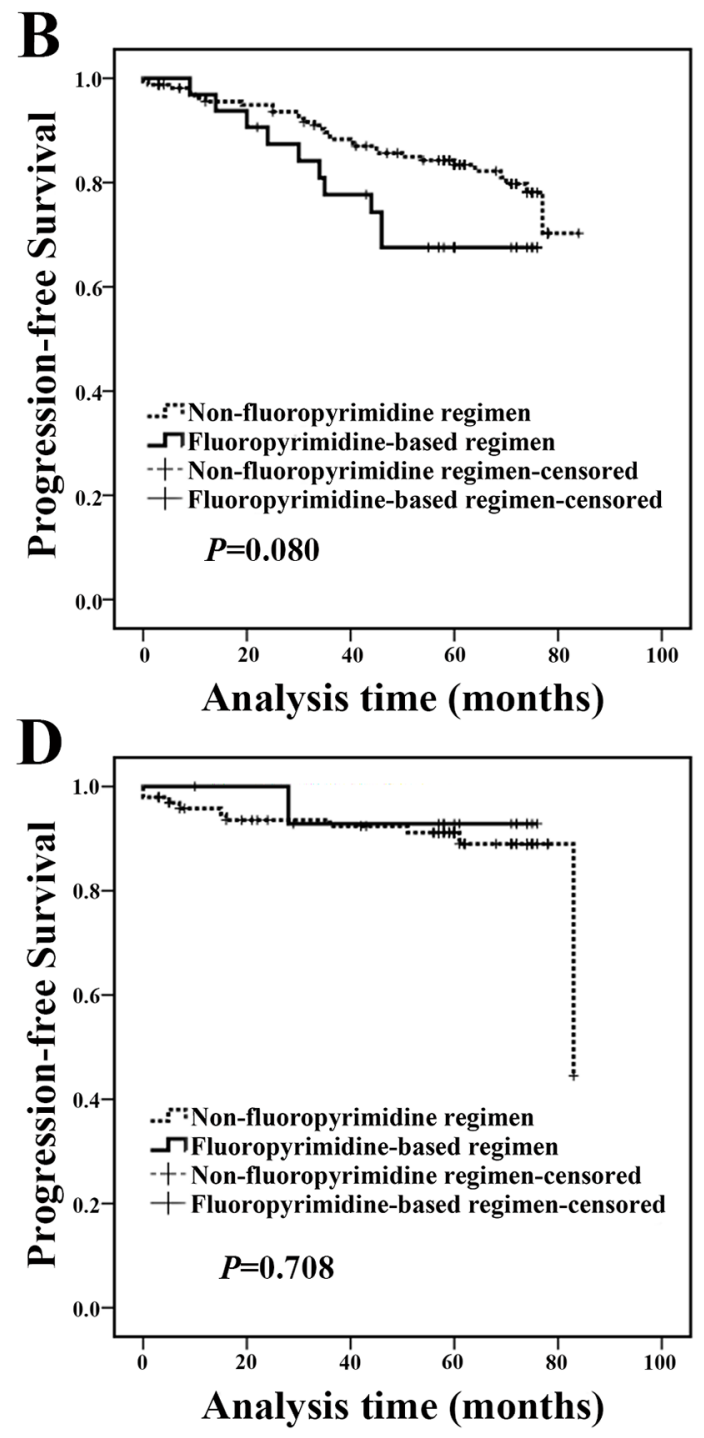

Figure 2: Non-wild type $D P Y D$ carriers treated with fluoropyrimidine-based regimen exhibited a poor prognosis. (A-B) Non-wild type $D P Y D$ carriers treated with fluoropyrimidine-based regimen exhibited a shorter OS compared with those treated with nonfluoropyrimidine regimen. (C-D) Wild type DPYD carriers treated with fluoropyrimidine-based regimen exhibited a similar OS and PFS compared with those with non-fluoropyrimidine regimen. 

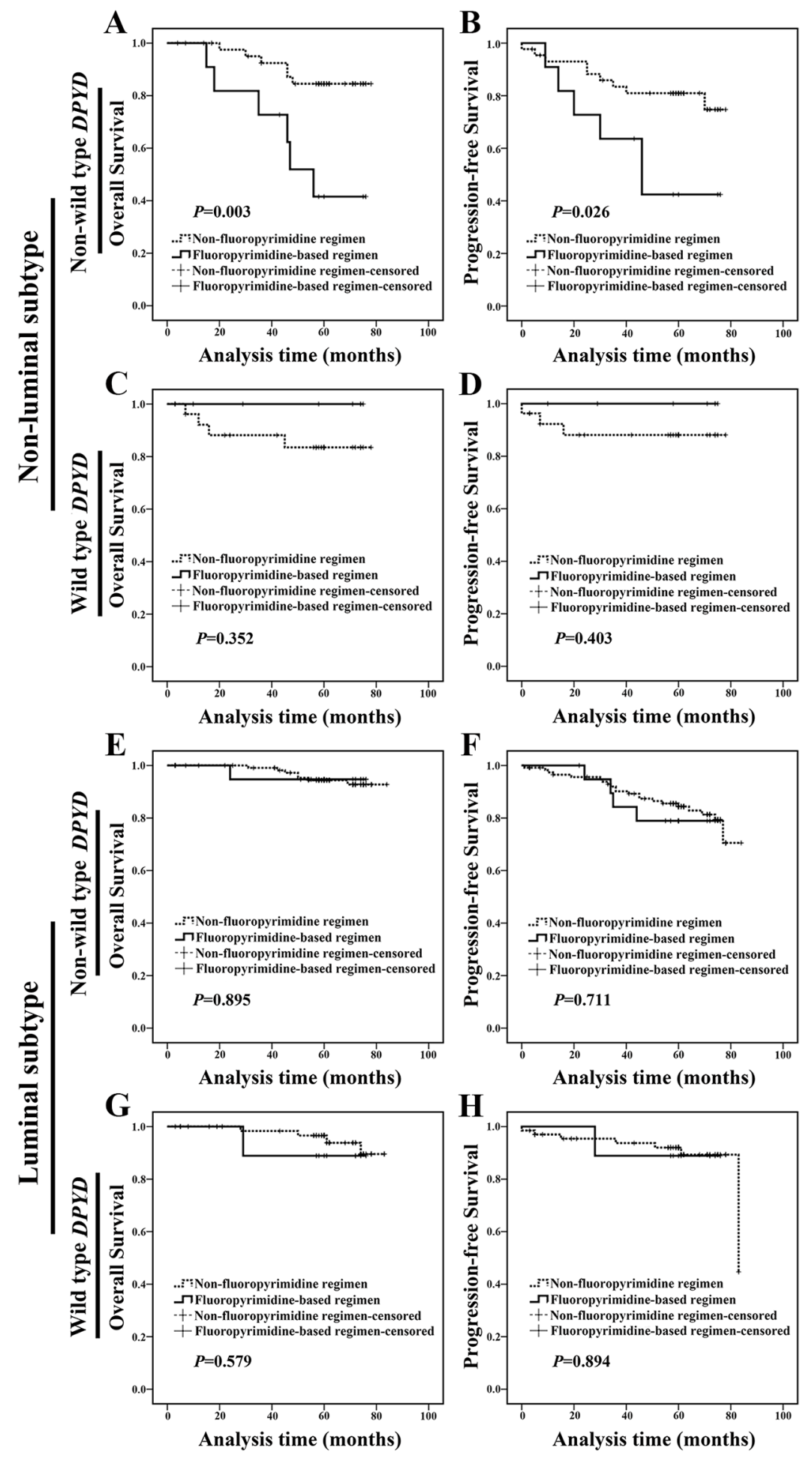

Figure 3: Non-wild type DPYD indicated a poor prognosis in non-luminal breast cancer patients treated with fluoropyrimidine-based regimen. (A-B) For non-luminal subtype, non-wild type DPYD carriers treated with fluoropyrimidinebased regimen exhibited a shorter OS and PFS compared with carriers treated with non-fluoropyrimidine regimen. (C-D) For non-luminal subtype, wild type DPYD carriers treated with fluoropyrimidine-based regimen exhibited a similar OS and PFS compared with those treated with non-fluoropyrimidine regimen. (E-F) For luminal subtype, non-wild type DPYD carriers treated with fluoropyrimidine-based regimen exhibited a similar OS and PFS compared with those treated with non-fluoropyrimidine regimen. (G-H) For luminal subtype, wild type DPYD carriers treated with fluoropyrimidine-based regimen exhibited a similar OS and PFS compared with those treated with nonfluoropyrimidine regimen. 
Meanwhile, Western blot analyses also were employed by using IDC tissues ( 8 cases carrying c. $1627 \mathrm{~A}>\mathrm{G}$ AA genotype and 8 cases carrying c. $1627 \mathrm{~A}>\mathrm{G}$ AG/GG genotype) and 2 primary cells (121918: c. $1627 \mathrm{~A}>\mathrm{G}$ AA; 1028: c. $1627 \mathrm{~A}>\mathrm{G} \mathrm{AG}$ ) derived from two non-luminal breast cancer patients (details in Table 3). Our results showed that the c. $1627 \mathrm{~A}>\mathrm{G} \mathrm{AG} / \mathrm{GG}$ had no effect on protein expression (Supplementary Figure 4).
In order to confirm our above conclusion, survival analysis of another cohort of 123 non-luminal subtype patients including 54 patients with c. $1627 \mathrm{~A}>\mathrm{G}$ AG/GG genotype and 69 patients with c.1627A $>$ G AA genotype were performed. For those c. $1627 \mathrm{~A}>\mathrm{G} \mathrm{AG} / \mathrm{GG}$ genotype carriers, patients treated with TE-based chemotherapy exhibited a better prognosis compared with those treated with fluoropyrimidines chemotherapy (OS: $P=0.007$,
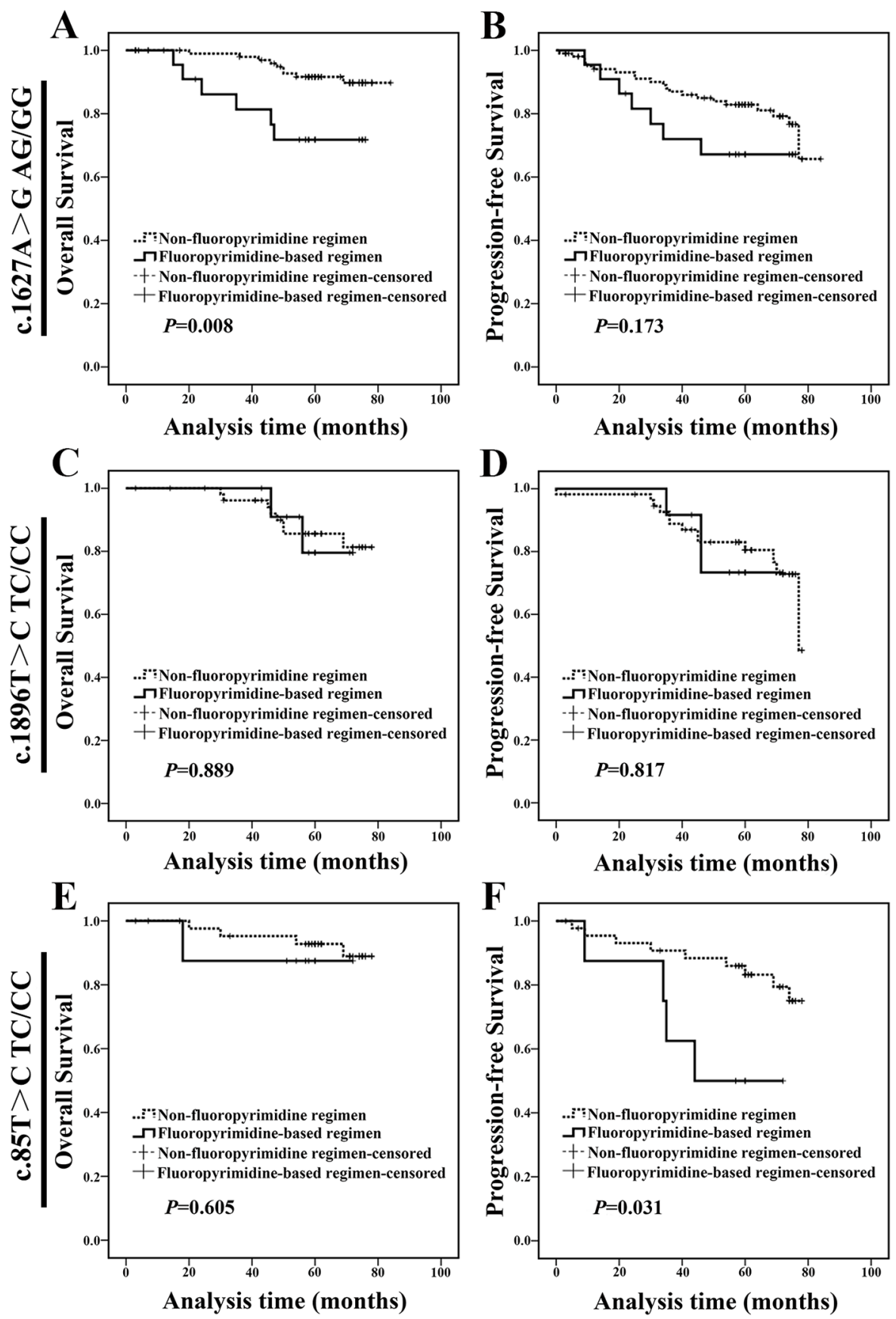

Figure 4: c.1627A>G AG/GG genotype carriers treated with fluoropyrimidine-based regimen exhibited a worse prognosis. (A-B) c.1627A $>$ G AG/GG genotype carriers treated with fluoropyrimidine-based regimen exhibited a shorter OS than those treated with non-fluoropyrimidine regimen. (C-D) c.1896T $>$ C TC/CC genotype carriers treated with fluoropyrimidine-based regimen exhibited a similar OS and PFS compared with those treated with non-fluoropyrimidine regimen. (E-F) c.85T $>$ C TC/CC genotype carriers treated with fluoropyrimidine-based regimen exhibited a similar OS compared with those treated with non-fluoropyrimidine regimen. 
Non-luminal subtype
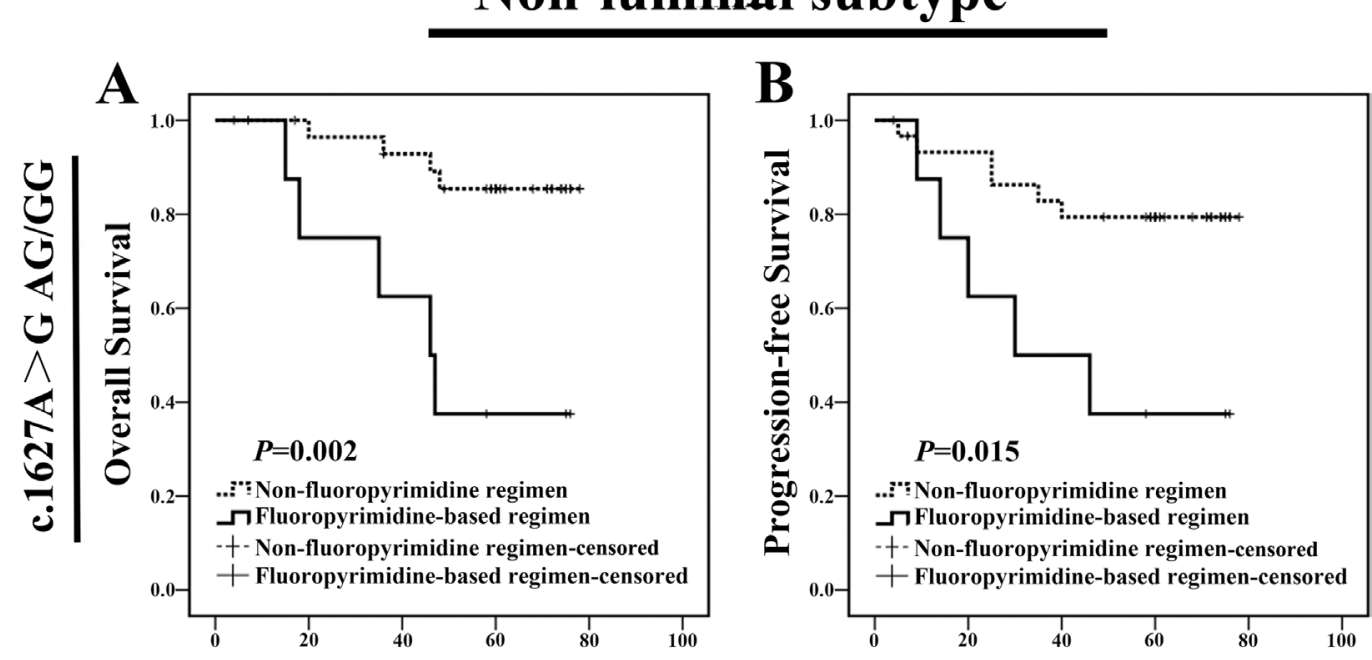

Analysis time (months)
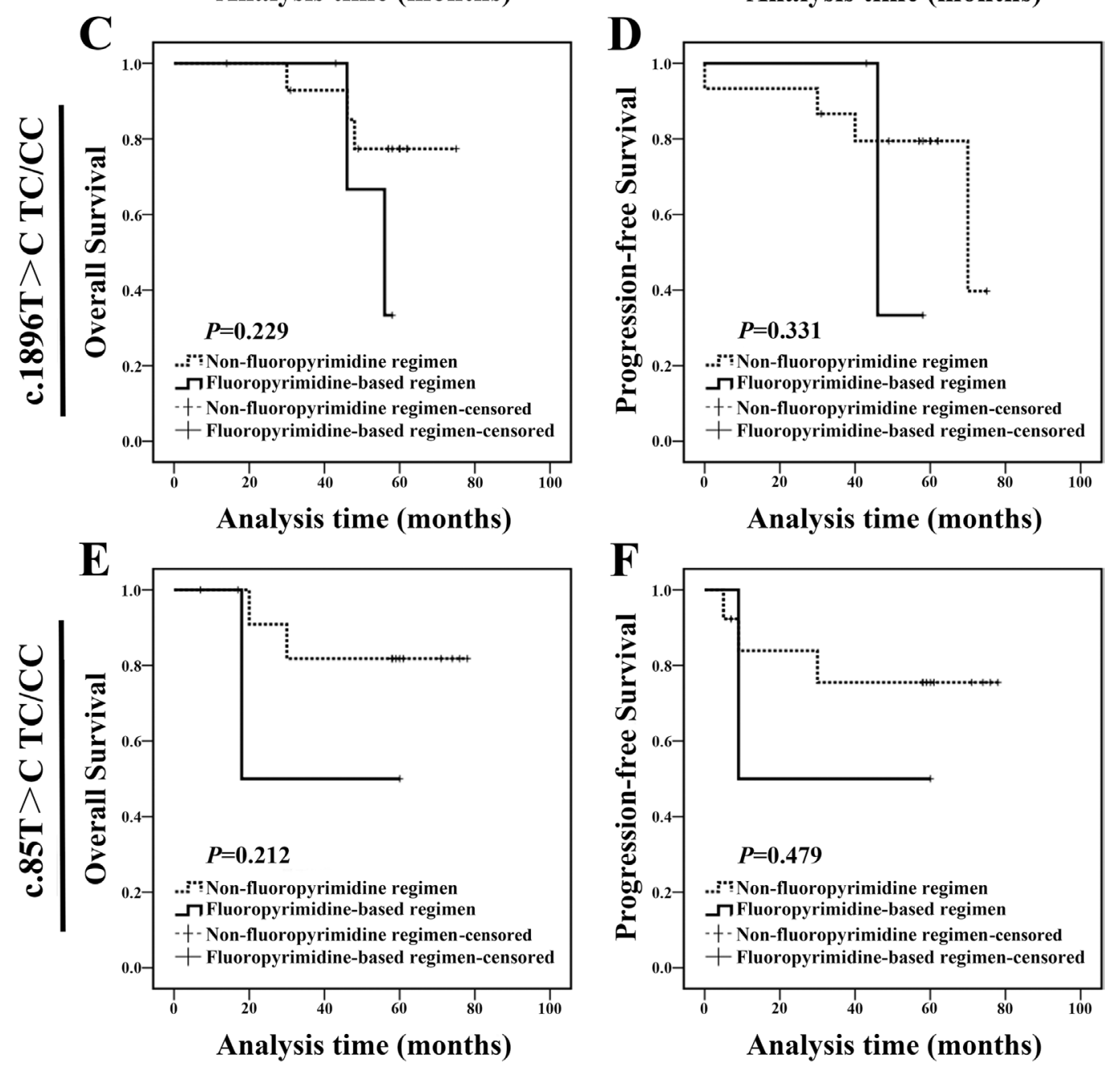

Figure 5: Non-luminal c.1627A>G AG/GG genotype carriers treated with fluoropyrimidine-based regimen exhibited a poor prognosis. (A-B) For non-luminal subtype, c. $1627 \mathrm{~A}>\mathrm{G} \mathrm{AG} / \mathrm{GG}$ genotype carriers treated with fluoropyrimidine-based regimen exhibited a shorter OS and PFS than those treated with non-fluoropyrimidine regimen. (C-D) For non-luminal subtype, c.1896T>C TC/ CC genotype carriers treated with fluoropyrimidine-based regimen exhibited a similar OS and PFS compared with those treated with nonfluoropyrimidine regimen. (E-F) For non-luminal subtype, c.85T $>\mathrm{C} \mathrm{TC/CC}$ genotype carriers treated with fluoropyrimidine-based regimen exhibited a similar OS and PFS compared with those with non-fluoropyrimidine regimen. 
PFS: $P=0.090$, Figure 9A-9B), and we did not find any difference in clinicopathologic characteristics between two populations at baseline (Supplementary Table 9). Meanwhile, for those c. $1627 \mathrm{~A}>\mathrm{G}$ AA genotype carriers, patients treated with fluoropyrimidines chemotherapy exhibited a similar survival with those treated with TEbased chemotherapy (Figure 9C-9D). All these results indicated that TE-based chemotherapy was a suitable regimen for non-luminal patients with DPYD c.1627A>G
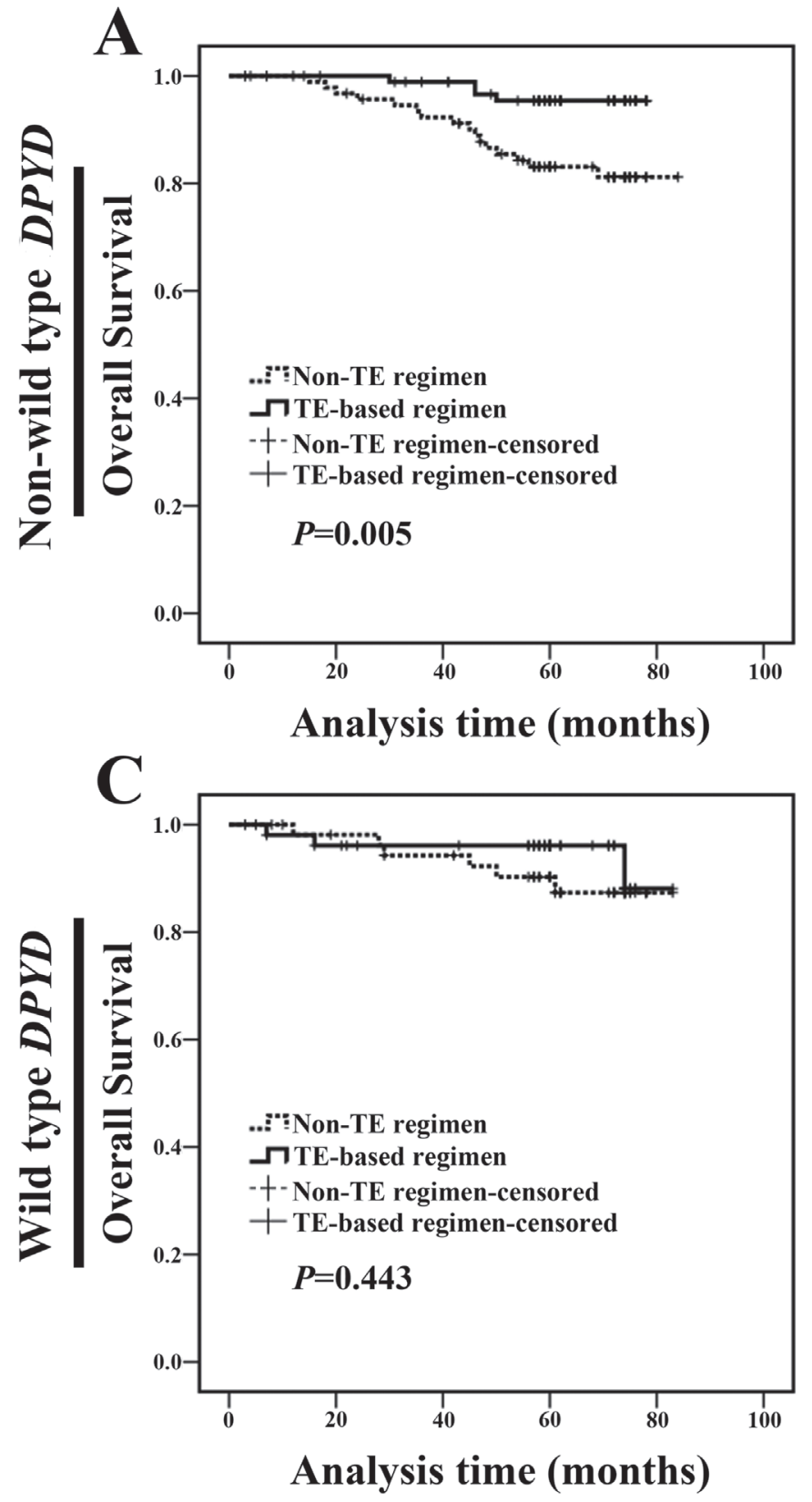

AG/GG genotype and fluoropyrimidine-based regimen should not be recommended for those patients.

Finally, both 121918 and 1028 primary cells were used for MTT assays to confirm above conclusion. After 5-Fu treatment, 1028 (c.1627A $>$ G AG) exhibited a higher cell viability than 121918 (c. $1627 \mathrm{~A}>\mathrm{G}$ AA) $(P=0.026$, Figure 10A). Meanwhile, 1028 exhibited a lower cell viability than 121918 with epirubicin $(P=0.045$, Figure $10 \mathrm{~B})$ or paclitaxel treatment $(P=0.012$, Figure 10C).
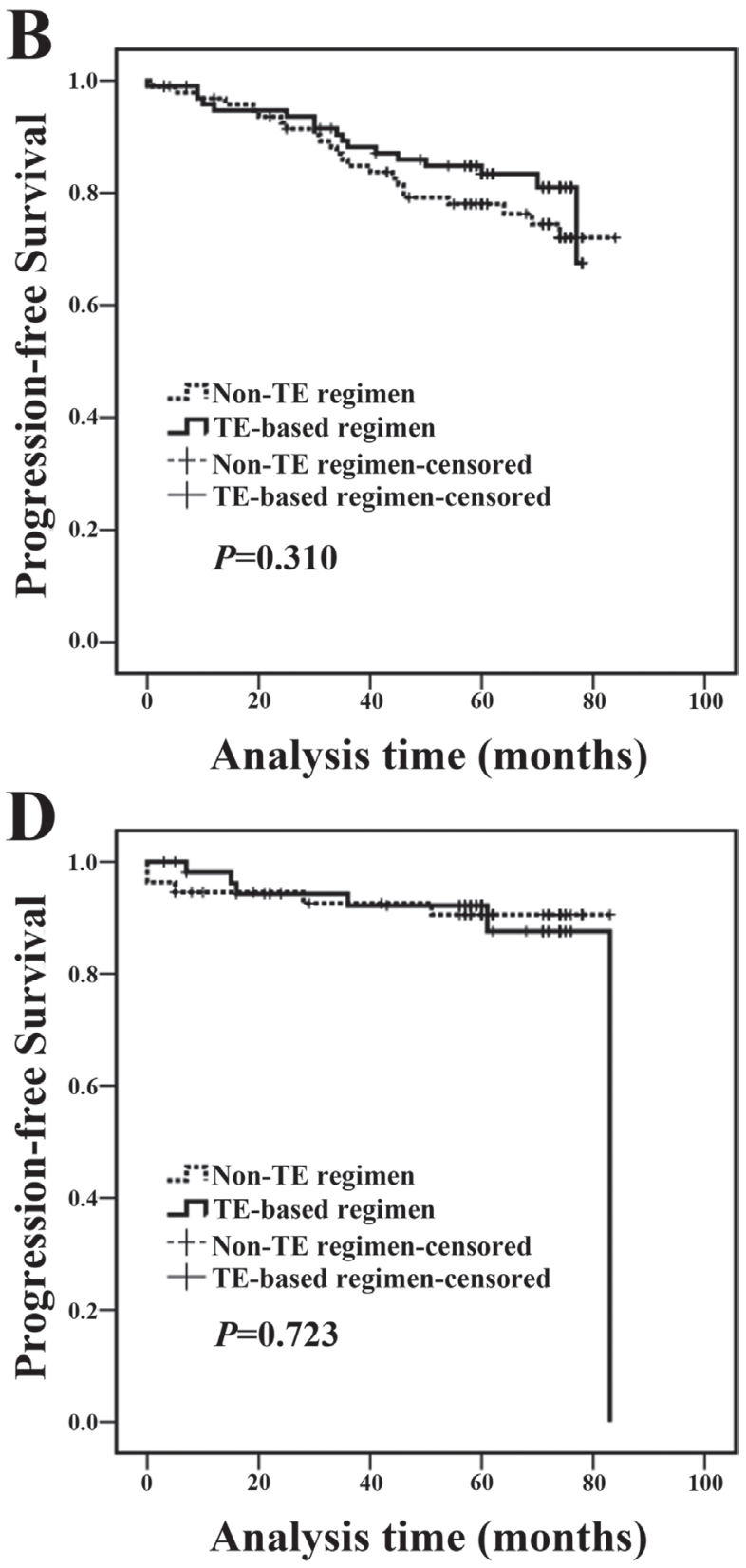

Figure 6: Non-wild type DPYD indicated a better prognosis in breast cancer patients treated with TE-based regimen. (A-B) Non-wild type DPYD carriers treated with TE-based regimen exhibited a longer OS compared with those treated with non-TE regimen. (C-D) Wild type DPYD carriers treated with TE-based regimen exhibited a similar OS and PFS compared with those treated with non-TE regimen. 

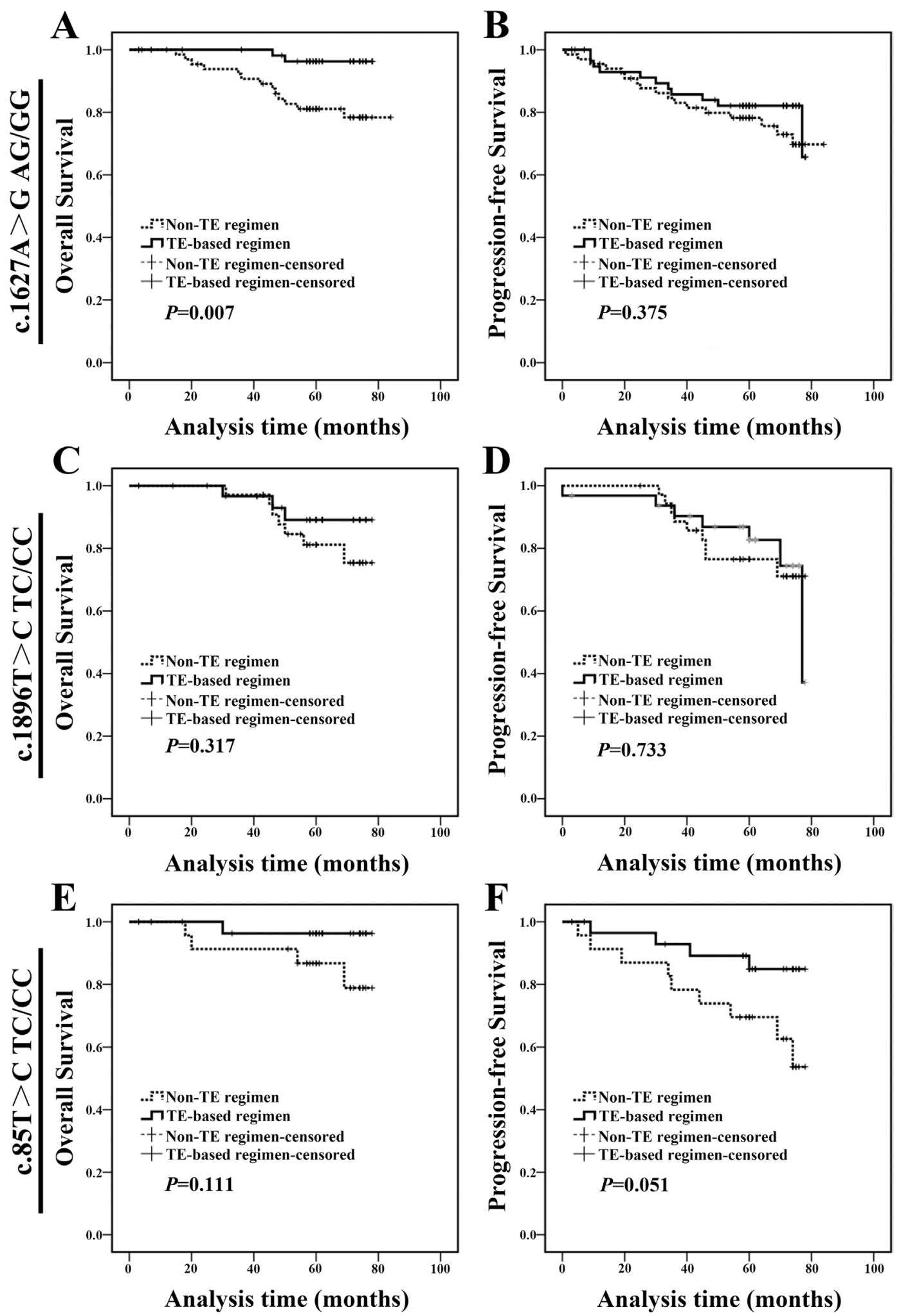

Figure 7: c.1627A $>$ G AG/GG genotype breast cancer patients treated with TE-based regimen exhibited a better prognosis. (A-B) c.1627A $>\mathrm{G}$ AG/GG genotype carriers treated with TE-based regimen exhibited a longer OS than those treated with non-TE regimen. (C-D) c.1896T>C TC/CC genotype carriers treated with TE-based regimen exhibited a similar OS and PFS with those treated with non-TE regimen. (E-F) c.85T $>\mathrm{C}$ TC/CC genotype carriers treated with TE-based regimen exhibited a similar OS and PFS compared with those treated with non-TE regimen. 
Table 3: Details of 2 primary breast cancer cell lines

\begin{tabular}{lccccccccc}
\hline $\begin{array}{l}\text { Primary } \\
\text { cell line }\end{array}$ & Gender & $\begin{array}{c}\text { Age } \\
\text { (years) }\end{array}$ & $\begin{array}{c}\text { Histological } \\
\text { grade }\end{array}$ & pTNM & $\begin{array}{c}\text { ER } \\
\text { status }\end{array}$ & $\begin{array}{c}\text { PR } \\
\text { status }\end{array}$ & $\begin{array}{c}\text { HER2 } \\
\text { status }\end{array}$ & $\begin{array}{c}\text { Molecular } \\
\text { subtype }\end{array}$ & $\begin{array}{c}\text { DP.1627A }>\text { G } \\
\text { genotype }\end{array}$ \\
\hline 121918 & female & 52 & III & II & Negative & Negative & Negative & $\begin{array}{c}\text { Triple negative } \\
\text { subtype }\end{array}$ \\
1028 & female & 64 & III & II & Negative & Negative & Positive & $\begin{array}{c}\text { HER2- } \\
\text { overexpression } \\
\text { subtype }\end{array}$ \\
\hline
\end{tabular}
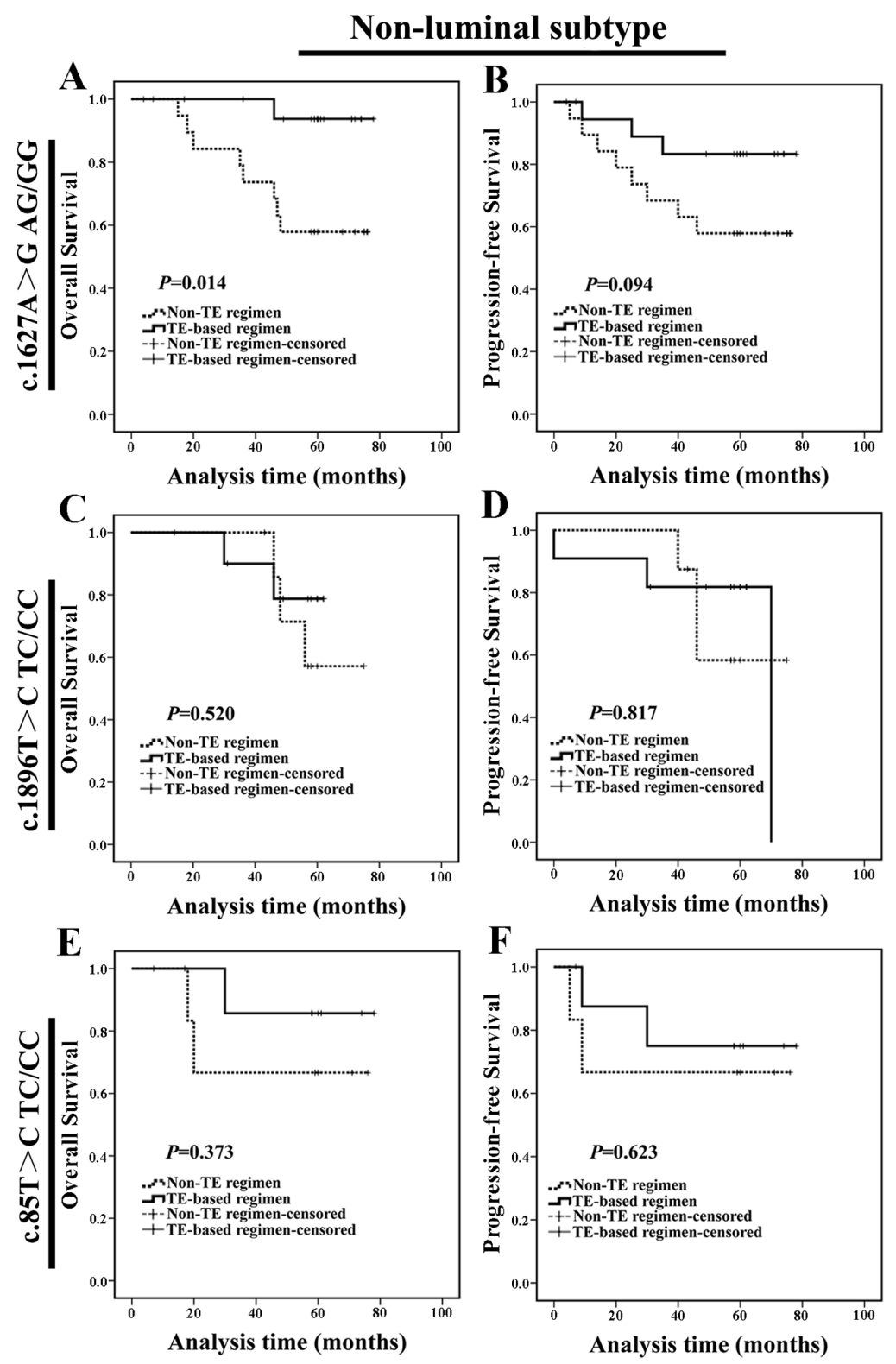

Figure 8: Non-luminal c.1627A $>$ G AG/GG genotype carriers treated with TE-based regimen exhibited a better prognosis. (A-B) For non-luminal subtype, c.1627A $>$ G AG/GG genotype carriers treated with TE-based regimen exhibited a longer OS than those treated with non-TE regimen. (C-D) For non-luminal subtype, c.1896T $>$ C TC/CC genotype carriers treated with TE-based regimen exhibited a similar OS and PFS compared with those treated with non-TE regimen. (E-F) For non-luminal subtype, c.85T $>\mathrm{C}$ TC/CC genotype carriers treated with TE-based regimen exhibited a similar OS and PFS compared with those treated with non-TE regimen. 


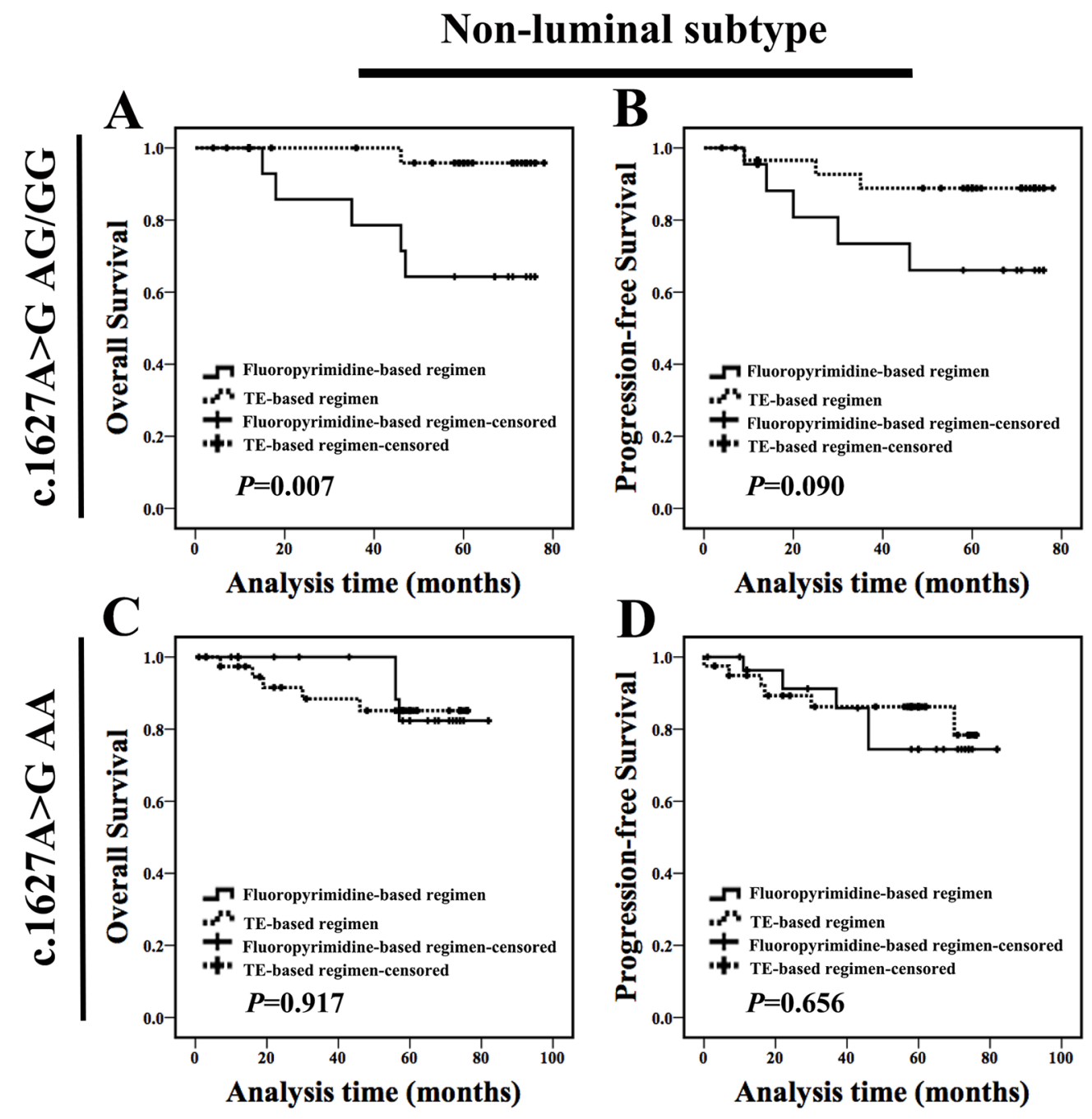

Figure 9: Non-luminal c.1627A>G AG/GG genotype carriers treated with fluoropyrimidines chemotherapy exhibited worse prognosis compared with those treated with TE-based regimen. (A-B) For non-luminal subtype, c.1627A $>$ G AG/GG genotype carriers treated with TE-based regimen exhibited a longer OS than those treated with fluoropyrimidines chemotherapy. (C-D) For non-luminal subtype, c.1627A $>$ G AA genotype carriers treated with TE-based regimen exhibited a similar OS and PFS compared with those treated with fluoropyrimidines chemotherapy.
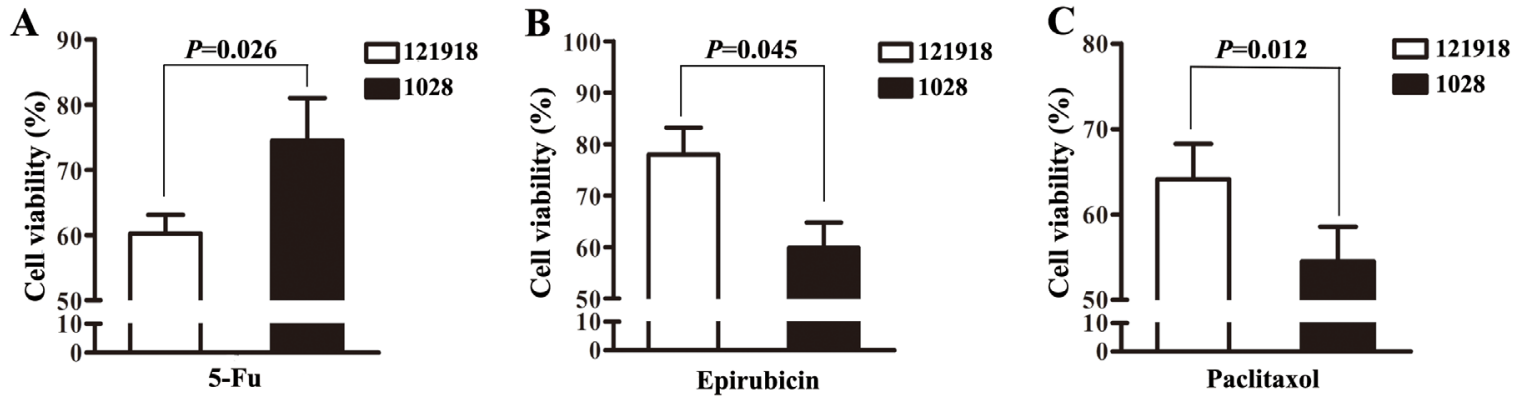

Figure 10: The effect of 5-Fu, epirubicin and paclitaxol on the growth of non-luminal breast cancer derived cells. (A) After primary cells were treated with $5-\mathrm{Fu}(1 \mu \mathrm{g} / \mathrm{ml})$ for $48 \mathrm{~h}$, cell viability of $121918(\mathrm{c} .1627 \mathrm{~A}>\mathrm{G} \mathrm{AA})$ and $1028(\mathrm{c} .1627 \mathrm{~A}>\mathrm{G}$ AG) was measured by MTT assay. (B) After primary cells were treated with epirubicin $(1 \mu \mathrm{g} / \mathrm{ml})$ for $48 \mathrm{~h}$, cell viability of 121918 and 1028 was measured by MTT assay. (C) After primary cells were treated with paclitaxol $(1 \mu \mathrm{g} / \mathrm{ml})$ for $48 \mathrm{~h}$, cell viability of 121918 and 1028 was measured by MTT assay. 


\section{DISCUSSION}

Although more than 160 polymorphisms in DPYD gene have been identified to date, data derived from Chinese cancer patients is very limited [19,20]. In the present study, we detected 5 polymorphisms including c. $74 \mathrm{~A}>\mathrm{G}, \quad$ c. $85 \mathrm{~T}>\mathrm{C}, \quad$ c. $1627 \mathrm{~A}>\mathrm{G}, \quad$ c. $1896 \mathrm{~T}>\mathrm{C}$ and c. $2194 \mathrm{G}>\mathrm{A}$ in breast cancer patients of China north area; and the genotype frequencies of these polymorphisms in our study were similar to previous research $[21,22]$. Furthermore, our research investigated the prognostic value of these polymorphisms for the first time in a large breast cancer patient population. We demonstrated for the first time that, $D P Y D$ gene polymorphisms could directly provide valuable prognostic information. We proposed for the first time that, TE-based chemotherapy was a suitable regimen for non-luminal breast cancer patients with $D P Y D$ c. $1627 \mathrm{~A}>\mathrm{G}$ AG/GG, whereas fluoropyrimidine-based chemotherapy should not be recommended.

Actually, previous studies on $D P Y D$ were mainly focused on $D P Y D$ polymorphisms-induced severe toxicities and tried to explain this phenomenon by deregulated DPD enzyme activity [23]. Unfortunately, prior investigations did not reach agreements and some researchers even got controversial conclusion. For example, Deenen et al. failed to show any correlation between c.85T $>\mathrm{C}, \quad$ c.1627A $>\mathrm{G}, \quad$ c.1896T $>\mathrm{C}$ and fluoropyrimidine-related toxicity in colorectal cancer patients [24], whereas in other reports, a statistically higher incidence of side effects was observed in gastrointestinal malignancies with those 3 SNPs listed above [14, 25]. In this study, we found no obvious fluoropyrimidinesassociated toxicity in breast cancer patients with non-wild type DPYD.

According to our results, non-luminal breast cancer patients carrying $D P Y D$ c. $1627 \mathrm{~A}>\mathrm{G} \mathrm{AG} / \mathrm{GG}$ treated with fluoropyrimidine-based regimen presented a shorter OS $(P=0.002)$ and PFS $(P=0.015)$ (Figure 5A and 5B). These results were somewhat indirectly supported by previous study in which fluorouracil-based chemotherapy showed no effect in gastric cancer patients carrying c. $1627 \mathrm{~A}>\mathrm{G}$ AG/GG genotype [16]. Furthermore, Gross's research demonstrated that somatic copy number changes in DPYD, rather than aberrant DPD protein level, could reflect a distinct tumor profile associated with distinct outcomes in breast cancer [26].

In our retrospectively study, we were unable to assess DPD enzyme activity since it was extremely complex to collect samples of subjects. Furthermore, given the ambiguous role of $\mathrm{c} .1627 \mathrm{~A}>\mathrm{G}$ polymorphisms in DPD enzyme activity based on previous studies and the complexity of DPD pharmacokinetics, we suggest that screening of patients for DPYD c. $1627 \mathrm{~A}>\mathrm{G}$ polymorphisms prior to administration of fluoropyrimidines will allow more appropriate and individualized approach to chemotherapy management.
The most clinically useful aspect of our results is the potential ability to identify patients with breast cancer who are insensitive to fluoropyrimidine-based regimen and likely to benefit from TE-based chemotherapy. As is well known, TE-based chemotherapy, including anthracycline and docetaxel, is widely used in breast cancer treatment. Anthracyclines are antibodies produced from the streptomyces species, which can inhibit nuclear enzyme TOPO (topoisomerase) II leading to DNA doublestrand breaks and cell death [27]. Docetaxel has the ability to polymerize tubulin in the absence of GTP, which under normal conditions is an absolute requirement for microtubule polymerization. Paclitaxel-bound microtubules are unusually stable and resistant to depolymerization. The most significant cellular impact of the interference with microtubule dynamics is during the mitotic phase of the cell cycle where paclitaxel inhibits mitotic spindle dynamics leading to mitotic arrest and consequent apoptotic cell death [27]. Although the mechanism linking DPYD SNPs and TE-based chemotherapy response is unknown and further exploration is necessary, we speculate that accumulation of DNA damage and abnormal DNA repair might contribute to this phenomenon.

In conclusion, our study demonstrated that TE-based chemotherapy was a suitable regimen for non-luminal breast cancer patients with $D P Y D$ c. $1627 \mathrm{~A}>\mathrm{G} \mathrm{AG} / \mathrm{GG}$, while fluoropyrimidine-based chemotherapy should not be recommended. Our findings provided a novel strategy, which will guide clinicians to choose more precise chemotherapy treatment for breast cancer patients.

\section{MATERIALS AND METHODS}

\section{Patient selection and clinical information}

Paraffin-embedded specimens of 331 breast cancer patients with invasive carcinoma, diagnosed between 2010 and 2011 were reviewed and randomly selected from Department of Breast Cancer Pathology and Research Laboratory, Tianjin Medical University Cancer Institute \& Hospital (Tianjin, China). The histopathology was reviewed and diagnosis of each case was confirmed independently by two pathologists according to World Health Organization (WHO) criteria. This study was approved by the Institutional Ethic Committee of Tianjin Medical University Cancer Institute \& Hospital (bc2016030). All experiments were performed in accordance with relevant guidelines and regulations of Ethic Committee of Tianjin Medical University Cancer Institute \& Hospital. All the patients signed informed consent for participation of the study and the use of their biological tissues.

331 patients were women aging from 23 to 89 years (mean 52.6 years) without preoperative radiation. Among them, 231 patients were luminal subtype, 51 patients were HER2-overexpression subtype and 44 were triple-negative subtype. 157 patients received TE (taxane 
and anthracycline) chemotherapies, 49 patients received chemotherapies with fluoropyrimidines and 70 patients received CET (cyclophosphamide, anthracycline and taxane) chemotherapy, chemotherapeutic information of 20 patients was missing, the rest (35 patients) were treated with other chemotherapy after operation. None patients presented severe toxic and side reaction of chemotherapy. A total of 320 cases were included for prognostic analyses, excluding 11 cases with no follow-up data. The follow-up was 3-84 months (Median: 58.3). Recurrences were recorded for 5 cases and 36 developed distant metastasis (bone metastasis: 13, lung metastasis: 16, liver metastasis: 9 , brain metastasis: 3, kidney metastasis: 3 , thyroid metastasis: 1 ). It was worth noting that multiple organic metastases were noted in 13 patients. 33 patients died during the follow-up period.

To further investigate our conclusion, another cohort of 123 non-luminal subtype breast cancer patients with follow-up (mean aged 52.61, range: 23-89) were selected. Among them, 52 patients received fluoropyrimidines and 71 patients received TE-based chemotherapies. Genomic DNA was isolated from tumor tissues using standard techniques and PCR-sequencing was applied to detect the SNPs status of c.1627A $>$ G. In our survival analysis, they were divided into four groups: patients with c.1627A $>\mathrm{G}$ AG/GG treated with fluoropyrimidines chemotherapy $(\mathrm{n}=23)$; patients with c. $1627 \mathrm{~A}>\mathrm{G}$ AG/GG treated with TE-based chemotherapy $(n=31)$; patients with c. $1627 \mathrm{~A}>\mathrm{G}$ AA treated with fluoropyrimidines chemotherapy $(n=29)$ and patients with c. $1627 \mathrm{~A}>\mathrm{G}$ AA treated with TE-based chemotherapy $(n=40)$.

16 IDC tissues ( 8 cases with c. $1627 \mathrm{~A}>\mathrm{G}$ AA and 8 cases with c. $1627 \mathrm{~A}>\mathrm{G} A \mathrm{G} / \mathrm{GG})$ were applied in the Western Blot assay. 2 primary breast cancer cells (121918 and 1028, derived from 2 non-luminal breast cancer patients, respectively) were applied in the Western Blot assay and MTT assay. 121918 cells was DPYD c. $1627 \mathrm{~A}>\mathrm{G}$ AA and 1028 cells was $D P Y D$ c. $1627 \mathrm{~A}>\mathrm{G}$ AG (details in Table 3). All patients were women without preoperative chemotherapy or radiation.

\section{The pathological diagnosis criteria of breast cancer molecular subtype}

Molecular subtype classification of breast cancer according to the St Gallen recommendation was based on the immunohistochemistry analysis of ER, PR and HER2 [28]. Luminal subtype (ER+ or/and PR+, HER2- or HER2+), non-luminal subtype (ER- and PR-, HER2- or HER2+). We provided representative immunohistochemical images of luminal and non-luminal subtype in Supplementary Figure 5.

\section{Determination of 5 DPYD SNPs status}

5 DPYD single nucleotide polymorphisms (SNPs) status (c.74A $>$ G, c.85T $>$ C, c. 1627A $>$ G, c.1896T $>$ C and c. $2194 \mathrm{G}>\mathrm{A}$ ) were determined using the polymerase chain reaction ( $\mathrm{PCR}$ )-sequencing method. The c.74A $>\mathrm{G}$ is a transition from $A$ to $G$ at nucleotide position 74, resulting to a Histidine change to an Arginine. The c.85T $>\mathrm{C}$ (rs1801265) is a transition from $\mathrm{T}$ to $\mathrm{C}$ at nucleotide position 85 with an amino acid change from Cysteine to Argnine. The c.1627A $>$ G (rs1801159) is a transition from A to $\mathrm{G}$ at nucleotide position 1627 , leading to an Isoleucine change to a Valine. The c.1896T $>\mathrm{C}$ (rs17376848) is a synonymous $\mathrm{SNP}$, which has a transition from $\mathrm{T}$ to $\mathrm{C}$ at nucleotide position 1896 . The c.2194G $>$ A ( $r$ 1801160) is a transition from $\mathrm{G}$ to $\mathrm{A}$ at nucleotide position 2194, leading to a Valine to an Isoleucine.

Genomic DNA was isolated from tumor tissues of invasive breast carcinoma patients using standard techniques. PCR-sequencing was applied to detect 5 SNPs status. In brief, 4 coding regions of DPYD (NM_000110.3) were amplified by using 4 polymerase chain reaction (PCR) mixtures respectively. DPYD c.74A $>\mathrm{G}$ and c. $85 \mathrm{~T}>\mathrm{C}$ were amplified in the same reaction, so 5 SNPs were amplified by 4 reaction mixtures. For the reaction of c.74A $>\mathrm{G}$ and c.85T $>\mathrm{C}$, PCR program was used as follows: initial denaturation $\left(94^{\circ} \mathrm{C}, 2 \mathrm{~min}\right)$; followed by 30 PCR cycles $\left(98^{\circ} \mathrm{C}, 20 \mathrm{~s} ; 70^{\circ} \mathrm{C}, 30 \mathrm{~s} ; 70^{\circ} \mathrm{C}, 40 \mathrm{~s}\right)$ and a final extension $\left(72^{\circ} \mathrm{C}, 10 \mathrm{~min}\right)$. For the reaction of c. $1627 \mathrm{~A}>\mathrm{G}, \mathrm{PCR}$ program was used as follows: initial denaturation $\left(94^{\circ} \mathrm{C}, 2 \mathrm{~min}\right)$; followed by 30 PCR cycles $\left(98^{\circ} \mathrm{C}, 20 \mathrm{~s} ; 52^{\circ} \mathrm{C}, 30 \mathrm{~s} ; 70^{\circ} \mathrm{C}, 40 \mathrm{~s}\right)$ and a final extension $\left(72^{\circ} \mathrm{C}, 10 \mathrm{~min}\right)$. For the reaction of c. $1896 \mathrm{~T}>\mathrm{C}, \mathrm{PCR}$ program was used as follows: initial denaturation $\left(94^{\circ} \mathrm{C}\right.$, $2 \mathrm{~min})$; followed by $30 \mathrm{PCR}$ cycles $\left(98^{\circ} \mathrm{C}, 20 \mathrm{~s} ; 59.1^{\circ} \mathrm{C}\right.$, $\left.30 \mathrm{~s} ; 70^{\circ} \mathrm{C}, 40 \mathrm{~s}\right)$ and a final extension $\left(72^{\circ} \mathrm{C}, 10 \mathrm{~min}\right)$. For the reaction of c. $2194 \mathrm{G}>\mathrm{A}, \mathrm{PCR}$ program was used as follows: initial denaturation $\left(94^{\circ} \mathrm{C}, 2 \mathrm{~min}\right)$; followed by 30 PCR cycles $\left(98^{\circ} \mathrm{C}, 20 \mathrm{~s} ; 56.1^{\circ} \mathrm{C}, 30 \mathrm{~s} ; 70^{\circ} \mathrm{C}, 40 \mathrm{~s}\right)$ and a final extension $\left(72^{\circ} \mathrm{C}, 10 \mathrm{~min}\right)$. Reactions were carried out in a total volume of $25 \mu \mathrm{l}$, using $100 \mathrm{ng}$ of genomic DNA as templates. After amplification, PCR products were sequenced by company (GENEWIZ, Suzhou, China). Primers for amplifications were all designed and synthesized by GENEWIZ, and primer sequences are presented in Supplementary Table 1.

\section{Statistical methods}

The SPSS 13.0 software package (SPSS, Chicago, IL, USA) was used for statistical analysis. The Chi-square $\left(\chi^{2}\right)$ test or Fisher's exact test was used for analysis of Hardy-Weinberg equilibrium and correlations between two variables were evaluated by Spearman rank correlation test. Progression-free survival (PFS) was defined as the time from surgery to recurrence or cancer-specific death, whichever occurred first. Overall survival (OS) was calculated from pathological diagnosis to the date of last contact or death from breast carcinoma. Survival analysis was performed according to the Kaplan-Meier method and assessed using the log-rank test. All statistical tests were 2 -tailed and $P<0.05$ was regarded as significant. 


\section{Western blot}

Tissues or cells were lysed in SDS lysis buffer on ice. Equal amounts of cell lysates were loaded and separated by SDS-PAGE, and proteins were transferred onto nitrocellulose membranes and incubated with the primary DPD antibody (1:1000, ab54797, abcam, USA) overnight at $4{ }^{\circ} \mathrm{C}$. Membranes were then treated with secondary antibodies. Blots were analyzed by Licor Odyssey infrared imaging.

\section{MTT assay}

$3 \times 10^{4}$ cells were seeded in $24-w e l l$ plates. After overnight culture to allow cells to adhere, culture medium containing 5-Fu, paclitaxel or epirubicin at indicated concentration was added for an additional $48 \mathrm{~h}$. Cells were then incubated with $500 \mu \mathrm{l}$ of the MTT stock solution $(5 \mathrm{mg} / \mathrm{ml})$ for 4 hours. Finally, medium was removed and the converted dye was solubilized with dimethyl sulfoxide. The absorbance of the converted dye was measured at a wave length of $570 \mathrm{~nm}$. The absorbance in the untreated control group was regarded as $100 \%$ cell viability.

\section{Author contributions}

Conception and design: Yongjie Ma, Feng Gu and Li Fu. Acquisition of data: Yong Huang, Huikun Zhang and Fengxia Qin. Performed the experiments: Yong Huang, Huikun Zhang and Fengxia Qin. Analysis of data: Yong Huang. Statistical analyses: Feng Yu and Limin Yang. Writing of manuscript: Fengxia Qin and Yongjie Ma. Preparation of tables and figures: Fengxia Qin and Xiaoli Liu. All authors reviewed the manuscript.

\section{CONFLICTS OF INTEREST}

The authors declare no conflicts of interest.

\section{GRANT SUPPORT}

This work was supported by National Scientific Foundation of China (81672636) from Dr. Feng Gu; National Scientific Foundation of China (81572851) from Dr. Yongjie Ma.

\section{REFERENCES}

1. Schmidt KT, Chau CH, Price DK, Figg WD. Precision Oncology Medicine: The Clinical Relevance of PatientSpecific Biomarkers Used to Optimize Cancer Treatment. J Clin Pharmacol. 2016; 56:1484-99.

2. Wei X, Duan W, Li Y, Zhang S, Xin X, Sun L, Gao M, Li Q, Wang D. AT101 exerts a synergetic efficacy in gastric cancer patients with 5-FU based treatment through promoting apoptosis and autophagy. Oncotarget. 2016; 7:34430-41. http://doi.org/10.18632/oncotarget.9119.
3. Tecza K, Pamula-Pilat J, Lanuszewska J, Grzybowska E. Genetic polymorphisms and response to 5-fluorouracil, doxorubicin and cyclophosphamide chemotherapy in breast cancer patients. Oncotarget. 2016; 7:66790-808. http://doi. org/10.18632/oncotarget.11053.

4. Patel K, Yerram SR, Azad NA, Kern SE. A thymidylate synthase ternary complex-specific antibody, FTS, permits functional monitoring of fluoropyrimidines dosing. Oncotarget. 2012; 3:678-85. http://doi.org/10.18632/ oncotarget.554.

5. Del Re M, Quaquarini E, Sottotetti F, Michelucci A, Palumbo R, Simi P, Danesi R, Bernardo A. Uncommon dihydropyrimidine dehydrogenase mutations and toxicity by fluoropyrimidines: a lethal case with a new variant. Pharmacogenomics. 2016; 17:5-9.

6. Lu ZH, Zhang R, Diasio RB. Purification and characterization of dihydropyrimidine dehydrogenase from human liver. J Biol Chem. 1992; 267:17102-9.

7. Milano G, Etienne MC. Dihydropyrimidine dehydrogenase (DPD) and clinical pharmacology of 5-fluorouracil (review). Anticancer Res. 1994; 14:2295-7.

8. Heggie GD, Sommadossi JP, Cross DS, Huster WJ, Diasio RB. Clinical pharmacokinetics of 5-fluorouracil and its metabolites in plasma, urine, and bile. Cancer Res. 1987; 47:2203-6.

9. Harris BE, Carpenter JT, Diasio RB. Severe 5-fluorouracil toxicity secondary to dihydropyrimidine dehydrogenase deficiency. A potentially more common pharmacogenetic syndrome. Cancer. 1991; 68:499-501.

10. Tuchman M, Ramnaraine ML, O'Dea RF. Effects of uridine and thymidine on the degradation of 5-fluorouracil, uracil, and thymine by rat liver dihydropyrimidine dehydrogenase. Cancer Res. 1985; 45:5553-6.

11. Offer SM, Wegner NJ, Fossum C, Wang K, Diasio RB. Phenotypic profiling of DPYD variations relevant to 5-fluorouracil sensitivity using real-time cellular analysis and in vitro measurement of enzyme activity. Cancer Res. 2013; 73:1958-68.

12. Zhao XQ, Cao WJ, Yang HP, Yang XW, Tang P, Sun L, Gao X. DPYD gene polymorphisms are associated with risk and chemotherapy prognosis in pediatric patients with acute lymphoblastic leukemia. Tumour Biol. 2016; 37:10393-402.

13. Saif MW. Dihydropyrimidine dehydrogenase gene (DPYD) polymorphism among Caucasian and non-Caucasian patients with 5-FU- and capecitabine-related toxicity using full sequencing of DPYD. Cancer Genomics Proteomics. 2013; 10:89-92.

14. Joerger $\mathrm{M}$, Huitema $\mathrm{AD}$, Boot $\mathrm{H}$, Cats $\mathrm{A}$, Doodeman VD, Smits PH, Vainchtein L, Rosing H, Meijerman I, Zueger M, Meulendijks D, Cerny TD, Beijnen JH, et al. Germline TYMS genotype is highly predictive in patients with metastatic gastrointestinal malignancies receiving capecitabine-based chemotherapy. Cancer Chemother Pharmacol. 2015; 75:763-72. 
15. Kelemen LE, Terry KL, Goodman MT, Webb PM, Bandera EV, McGuire V, Rossing MA, Wang Q, Dicks E, Tyrer JP, Song H, Kupryjanczyk J, Dansonka-Mieszkowska A, et al. Consortium analysis of gene and gene-folate interactions in purine and pyrimidine metabolism pathways with ovarian carcinoma risk. Mol Nutr Food Res. 2014; 58:2023-35.

16. Zhang XP, Bai ZB, Chen BA, Feng JF, Yan F, Jiang Z, Zhong YJ, Wu JZ, Chen L, Lu ZH, Tong N, Zhang ZD, $\mathrm{Xu} \mathrm{PP}$, et al. Polymorphisms of dihydropyrimidine dehydrogenase gene and clinical outcomes of gastric cancer patients treated with fluorouracil-based adjuvant chemotherapy in Chinese population. Chin Med J (Engl). 2012; 125:741-6.

17. Zeng H, Yu H, Lu L, Jain D, Kidd MS, Saif MW, Chanock SJ, Hartge P, PanScan C, Risch HA. Genetic effects and modifiers of radiotherapy and chemotherapy on survival in pancreatic cancer. Pancreas. 2011; 40:657-63.

18. Trudeau M, Charbonneau F, Gelmon K, Laing K, Latreille J, Mackey J, McLeod D, Pritchard K, Provencher L, Verma S. Selection of adjuvant chemotherapy for treatment of node-positive breast cancer. Lancet Oncol. 2005; 6:886-98.

19. Toffoli G, Giodini L, Buonadonna A, Berretta M, De Paoli A, Scalone S, Miolo G, Mini E, Nobili S, Lonardi S, Pella N, Lo Re G, Montico M, et al. Clinical validity of a DPYDbased pharmacogenetic test to predict severe toxicity to fluoropyrimidines. Int J Cancer. 2015; 137:2971-80.

20. Huang L, Chen F, Chen Y, Yang X, Xu S, Ge S, Fu S, Chao T, Yu Q, Liao X, Hu G, Zhang P, Yuan X. Thymidine phosphorylase gene variant, platelet counts and survival in gastrointestinal cancer patients treated by fluoropyrimidines. Sci Rep. 2014; 4:5697.

21. Hsiao HH, Yang MY, Chang JG, Liu YC, Liu TC, Chang CS, Chen TP, Lin SF. Dihydropyrimidine dehydrogenase pharmacogenetics in the Taiwanese population. Cancer Chemother Pharmacol. 2004; 53:445-51.
22. He YF, Wei W, Zhang X, Li YH, Li S, Wang FH, Lin XB, Li ZM, Zhang DS, Huang HQ, Hu B, Jiang WQ. Analysis of the DPYD gene implicated in 5-fluorouracil catabolism in Chinese cancer patients. J Clin Pharm Ther. 2008; 33:307-14.

23. Yen JL, McLeod HL. Should DPD analysis be required prior to prescribing fluoropyrimidines? Eur J Cancer. 2007; 43:1011-6.

24. Deenen MJ, Tol J, Burylo AM, Doodeman VD, de Boer A, Vincent A, Guchelaar HJ, Smits PH, Beijnen JH, Punt CJ, Schellens JH, Cats A. Relationship between single nucleotide polymorphisms and haplotypes in DPYD and toxicity and efficacy of capecitabine in advanced colorectal cancer. Clin Cancer Res. 2011; 17:3455-68.

25. Sun W, Yan C, Jia S, Hu J. Correlation analysis of peripheral DPYD gene polymorphism with 5-fluorouracil susceptibility and side effects in colon cancer patients. Int $\mathrm{J}$ Clin Exp Med. 2014; 7:5857-61.

26. Gross E, Meul C, Raab S, Propping C, Avril S, Aubele M, Gkazepis A, Schuster T, Grebenchtchikov N, Schmitt M, Kiechle M, Meijer J, Vijzelaar R, et al. Somatic copy number changes in DPYD are associated with lower risk of recurrence in triple-negative breast cancers. Br J Cancer. 2013; 109:2347-55.

27. Crown J, Dieras V, Kaufmann M, von Minckwitz G, Kaye S, Leonard R, Marty M, Misset JL, Osterwalder B, Piccart M. Chemotherapy for metastatic breast cancer-report of a European expert panel. Lancet Oncol. 2002; 3:719-27.

28. Goldhirsch A, Wood WC, Coates AS, Gelber RD, Thürlimann B, Senn HJ. Strategies for subtypes-dealing with the diversity of breast cancer: highlights of the St. Gallen International Expert Consensus on the Primary Therapy of Early Breast Cancer 2011. Ann Oncol. 2011; 22:1736-47. 